\title{
Delay-sensitive and delay-insensitive deconvolution perfusion-CT: similar ischemic core and penumbra volumes if appropriate threshold selected for each
}

\author{
Fengyuan Man • James T. Patrie • Wenjun Xin • Guangming Zhu • Qinghua Hou • \\ Patrik Michel • Ashraf Eskandari • Tudor Jovin • Junfang Xian • Zhenchang Wang • \\ Max Wintermark
}

Received: 15 January 2015 / Accepted: 25 February 2015 / Published online: 7 March 2015

(C) Springer-Verlag Berlin Heidelberg 2015

\begin{abstract}
Introduction Perfusion-CT (PCT) processing involves deconvolution, a mathematical operation that computes the perfusion parameters from the PCT time density curves and an arterial curve. Delay-sensitive deconvolution does not correct for arrival delay of contrast, whereas delay-insensitive deconvolution does. The goal of this study was to compare delay-sensitive and delay-insensitive deconvolution PCT in terms of delineation of the ischemic core and penumbra.

Methods We retrospectively identified 100 patients with acute ischemic stroke who underwent admission PCT and CT angiography (CTA), a follow-up vascular study to determine recanalization status, and a follow-up noncontrast head CT (NCT) or MRI to calculate final infarct volume. PCT datasets were processed twice, once using delay-sensitive deconvolution and once using delay-insensitive deconvolution. Regions of interest (ROIs) were drawn, and cerebral blood flow (CBF), cerebral blood volume (CBV),
\end{abstract}

F. Man $\cdot$ J. Xian $\cdot$ Z. Wang

Department of Radiology, Beijing Tongren Hospital, Capital Medical

University, Beijing, China

F. Man $\cdot$ G. Zhu $\cdot$ Q. Hou $\cdot$ M. Wintermark

Department of Radiology, Neuroradiology Division, University of

Virginia, Charlottesville, VA, USA

\section{J. T. Patrie • W. Xin}

Department of Public Health Sciences, University of Virginia,

Charlottesville, VA, USA

G. Zhu

Department of Neurology, Military General Hospital of Beijing PLA,

Beijing, China

Q. Hou

Department of Neurology, The Second Affiliated Hospital of

Guangzhou Medical University, Guangzhou, China and mean transit time (MTT) in these ROIs were recorded and compared. Volume and geographic distribution of ischemic core and penumbra using both deconvolution methods were also recorded and compared.

Results MTT and CBF values are affected by the deconvolution method used $(p<0.05)$, while CBV values remain unchanged. Optimal thresholds to delineate ischemic core and penumbra are different for delay-sensitive (145\% MTT, CBV $\left.2 \mathrm{ml} \times 100 \mathrm{~g}^{-1} \times \mathrm{min}^{-1}\right)$ and delay-insensitive deconvolution (135\% MTT, CBV $2 \mathrm{ml} \times 100 \mathrm{~g}^{-1} \times \mathrm{min}^{-1}$ for delay-insensitive deconvolution). When applying these different thresholds, however, the predicted ischemic core $(p=0.366)$ and penumbra $(p=0.405)$ were similar with both methods. Conclusion Both delay-sensitive and delay-insensitive deconvolution methods are appropriate for PCT processing in acute ischemic stroke patients. The predicted ischemic core and penumbra are similar with both methods when using different sets of thresholds, specific for each deconvolution method.

\section{P. Michel · A. Eskandari}

Department of Neurology, Centre Hospitalier Universitaire Vaudois,

Lausanne, Switzerland

T. Jovin

Department of Neurology, University of Pittsburgh, Pittsburgh, PA, USA

M. Wintermark

Department of Radiology, Centre Hospitalier Universitaire Vaudois, Lausanne, Switzerland

M. Wintermark $(\bowtie)$

Department of Radiology, Neuroradiology Division, Stanford

University, 300 Pasteur Drive, Room S047,

Stanford, CA 94305-5105, USA

e-mail: Max.Wintermark@gmail.com 
Keywords Perfusion-CT · Deconvolution · Delay $\cdot$ Ischemic core $\cdot$ Ischemic penumbra

$\begin{array}{ll}\text { Abbreviations } \\ \text { PCT } & \text { Perfusion-CT } \\ \text { ASPECTS } & \text { Alberta stroke program early CT score } \\ \text { CBF } & \text { Cerebral blood flow } \\ \text { CBV } & \text { Cerebral blood volume } \\ \text { MTT } & \text { Mean transit time } \\ \text { ROI } & \text { Region of interest } \\ \text { NCT } & \text { Noncontrast head CT }\end{array}$

\section{Introduction}

Perfusion-CT (PCT) is an imaging technique used to assess cerebral perfusion [1]. PCT applications encompass acute stroke [2], chronic cerebrovascular conditions [3], vasospasm [4], traumatic brain injury [5], etc. Several processing methods exist to extract meaningful information from the PCT raw data $[6,7]$. The two main approaches include nondeconvolution models (or maximal slope models) or deconvolution models [8], the latter gaining more widespread acceptance recently [9]. The deconvolution operation is a complex mathematical operation that computes the perfusion parameters from the PCT time density curves and an arterial curve, in order to take into account the dispersion of the contrast bolus injected for the PCT study [10]. Deconvolution comes in several flavors [11-13], including delay-sensitive ones and delay-insensitive ones. Delaysensitive deconvolution does not correct for the arrival delay of the contrast between the selected arterial input function and the examined parenchymal voxel, whereas delayinsensitive deconvolution does [6]. Recently, some have suggested that in the setting of acute ischemic stroke, delay-insensitive deconvolution may lead to more accurate delineation of the ischemic core and especially penumbra, while delay-sensitive deconvolution methods may overestimate the penumbra [14].

The goal of this study was to compare delay-sensitive and delay-insensitive deconvolution PCT in terms of delineation of the ischemic core and penumbra.

\section{Material and methods}

Study patients

The clinical and imaging data presented in this study belongs to a repository created from the data collected as part of the standard clinical stroke care at three participating institutions, including the University of Virginia Health System, Charlottesville, VA; the Centre Hospitalier Universitaire Vaudois, Lausanne, Switzerland; and the University of Pittsburgh Medical Center, Pittsburgh, PA. Collection and analysis of data for and from the repository were approved by the respective institutional review boards of the three contributing institutions.

We retrospectively identified all consecutive patients included in this repository who met the following inclusion criteria: (1) acute ischemic stroke without evidence of intracerebral hemorrhage; (2) performance of a stroke CT work-up upon admission including PCT and CT angiography (CTA); (3) performance of a follow-up vascular study to determine recanalization status; and (4) of a follow-up noncontrast head CT (NCT) or MRI, which was used to calculate the final infarct volume on the slices matching the baseline PCT slices.

The following demographic and clinical variables were recorded: age, gender, time from symptom onset to baseline imaging, and time from onset to follow-up imaging.

\section{PCT image acquisition}

PCT studies were obtained on 16- and 64-slice CT scanners. Each PCT study involved successive gantry rotations performed in cine mode during intravenous administration of one or two boluses of 40-50 ml of iodinated contrast material at an injection rate of $4-5 \mathrm{ml} / \mathrm{s}$. Total PCT coverage ranged from 20 to $80 \mathrm{~mm}$, with 5- or 10-mm slices. Acquisition parameters were $80 \mathrm{kVp}$ and $100-200 \mathrm{mAs}$. Total PCT acquisition duration ranged from 50 to $70 \mathrm{~s}$. Sampling rate ranged from 1 to $2 \mathrm{~s}$ (Table 1 ).

PCT image post-processing and interpretation

PCT data was analyzed using Philips Brain Perfusion software version 4.5.2 (Philips Medical Systems, Cleveland, OH,

Table 1 Perfusion-CT (PCT) protocols used in patients included in the current study

\begin{tabular}{|c|c|c|c|c|}
\hline Scanner type & 16 slice & 16 slice & 64 slice & 64 slice \\
\hline Slice thickness & $10 \mathrm{~mm}$ & $5 \mathrm{~mm}$ & $5 \mathrm{~mm}$ & $5 \mathrm{~mm}$ \\
\hline $\begin{array}{l}\text { Total PCT } \\
\text { coverage } \\
\text { (all boluses) }\end{array}$ & $20 \mathrm{~mm}$ & $40 \mathrm{~mm}$ & $40 \mathrm{~mm}$ & $80 \mathrm{~mm}$ \\
\hline \multirow{2}{*}{$\begin{array}{l}\text { Acquisition } \\
\text { parameters }\end{array}$} & $80 \mathrm{kVp}$ & $80 \mathrm{kVp}$ & $80 \mathrm{kVp}$ & $80 \mathrm{kVp}$ \\
\hline & $200 \mathrm{mAs}$ & $100 \mathrm{mAs}$ & $100 \mathrm{mAs}$ & $100 \mathrm{mAs}$ \\
\hline $\begin{array}{l}\text { Total PCT } \\
\text { acquisition } \\
\text { duration }\end{array}$ & $50 \mathrm{~s}$ & $50 \mathrm{~s}$ & $70 \mathrm{~s}$ & $70 \mathrm{~s}$ \\
\hline Sampling rate & 1 per second & 1 per second & 1 per $2 \mathrm{~s}$ & $1-2$ per second \\
\hline $\begin{array}{c}\text { Number of } \\
\text { contrast } \\
\text { boluses }\end{array}$ & 1 & 2 & 1 & 2 \\
\hline Injection rate & $4 \mathrm{~cm}^{3} / \mathrm{s}$ & $5 \mathrm{~cm}^{3} / \mathrm{s}$ & $5 \mathrm{~cm}^{3} / \mathrm{s}$ & $5 \mathrm{~cm}^{3} / \mathrm{s}$ \\
\hline
\end{tabular}


USA), previously described [15]. This software is based on the central volume principle. Arterial input function and venous output selection is semi-automated and MTT maps are created using a closed-form deconvolution. Cerebral blood volume (CBV) values are calculated from the area under the time-density curves. Cerebral blood flow (CBF) is calculated as CBV divided by MTT. PCT was processed once using delay-sensitive deconvolution (singular value decomposition) and one using delay-insensitive deconvolution (blockcirculant singular value decomposition).

Regions of interest (ROIs) were drawn manually to include the cerebral hemisphere, the basal ganglia, and the white matter in the frontal and parietal lobes. $\mathrm{CBF}, \mathrm{CBV}$, and mean transit time (MTT) values in these ROIs were recorded separately for the ischemic hemisphere and the contralateral hemisphere.

For delay-sensitive deconvolution, PCT ischemic core and penumbra were delineated using validated MTT and CBV thresholds (MTT $>145 \%$ of the contralateral side values and CBV $<2.0 \mathrm{ml} / 100 \mathrm{~g}$ to define PCT ischemic core; MTT $>145 \%$ of the contralateral side values and CBV $>2.0 \mathrm{ml} /$ $100 \mathrm{~g}$ to define PCT ischemic penumbra) [16]. For delayinsensitive deconvolution, various combinations of MTT and CBV thresholds were tested (Table 2). The volumes of PCT ischemic core and penumbra, automatically measured by the software, were recorded, both for the delay-sensitive and the delay-insensitive approaches.

Both for delay-sensitive and delay-insensitive deconvolution, and for each set of MTT and CBV thresholds, the anatomical distribution of the ischemic core and penumbra were recorded using a modified Alberta Stroke Program Early CT score (ASPECTS) approach [17]. Each of the 10 ASPECT regions, plus the superior and inferior anterior cerebral artery territories, plus the thalamus and the posterior cerebral artery territory, were recorded either as normal, ischemic core ( $>50 \%$ occupied by ischemic core), or ischemic penumbra ( $>50 \%$ occupied by ischemic penumbra). An ischemic core score ranging from 0 (no ischemic core) to 14 (ischemic core occupying the whole hemisphere), and an ischemic penumbra score also ranging from 0 to 14 (but the sum of the two scores not exceeding 14), were calculated both for delay-sensitive and delay-insensitive deconvolution, and for each set of MTT and CBV thresholds.

\section{Statistical analysis}

Descriptive statistics Continuous data (CBF, CBV, and MTT values in ROIs) were summarized by the mean \pm standard deviation. Frequency data (e.g., gender) were summarized by counts.

Analysis \#1 The CBF, CBV, and MTT values measured in the ROIs using delay-sensitive and delay-corrected deconvolution were compared using Bland-Altman and linear regression analysis. Statistical significance was set at 0.05 .

Analysis \#2 The volumes of ischemic core and penumbra as calculated by delay-sensitive deconvolution were used as a reference standard. The volumes of ischemic core and penumbra calculated using delay-insensitive deconvolution and the different combinations of MTT and CBV thresholds were evaluated to determine the MTT/CBV combination for which the delay-insensitive core and penumbra volumes showed the strongest linear regression with the delaysensitive core and penumbra volumes, used as reference standard.

Analysis \#3 The modified ASPECT scores for ischemic core and penumbra obtained from delay-sensitive and delayinsensitive deconvolution were dichotomized and compared to each other using McNemar chi-square tests. Statistical significance was set at 0.05 .

Analysis \#4 Both for delay-sensitive and delay-insensitive deconvolution, the ischemic core volume was compared to the final infarct volume in the patients showing recanalization; and the ischemic core + penumbra volume was compared to the final infarct volume in the patients showing persistent arterial occlusion. Comparisons were performed using linear regression analysis.
Table 2 Online only. Various combinations of MTT and CBV thresholds were tested

\begin{tabular}{lllllll}
\hline \multicolumn{7}{c}{ MTT } \\
\cline { 3 - 7 } CBV & \multicolumn{7}{c}{$>125 \%$} & $>135 \%$ & $>145 \%$ & $>155 \%$ & $>165 \%$ \\
\hline Ischemic penumbra & $>1.8 \mathrm{ml} / 100 \mathrm{~g}$ & $\checkmark$ & $\checkmark$ & $\checkmark$ & & \\
& $>1.9 \mathrm{ml} / 100 \mathrm{~g}$ & & $\checkmark$ & $\checkmark$ & $\checkmark$ & $\checkmark$ \\
Ischemic core & $>2.0 \mathrm{ml} / 100 \mathrm{~g}$ & & $\checkmark$ & $\checkmark$ & $\checkmark$ & $\checkmark$ \\
& $<1.8 \mathrm{ml} / 100 \mathrm{~g}$ & $\checkmark$ & $\checkmark$ & $\checkmark$ & & $\checkmark$ \\
& $<1.9 \mathrm{ml} / 100 \mathrm{~g}$ & & $\checkmark$ & $\checkmark$ & $\checkmark$ & $\checkmark$ \\
& $<2.0 \mathrm{ml} / 100 \mathrm{~g}$ & & $\checkmark$ & $\checkmark$ & $\checkmark$ & $\checkmark$ \\
\hline
\end{tabular}


Table 3 Mean \pm standard deviation of CBF and MTT values obtained with delay-sensitive and delay-insensitive deconvolution. 84/86/106

\begin{tabular}{|c|c|c|c|c|c|}
\hline \multirow[t]{2}{*}{ Variable } & \multirow[t]{2}{*}{ Location } & \multicolumn{2}{|l|}{ Ischemic side } & \multicolumn{2}{|l|}{ Nonischemic side } \\
\hline & & Delay-sensitive & Delay-insensitive & Delay-sensitive & Delay-insensitive \\
\hline \multirow[t]{3}{*}{$\mathrm{CBF}\left(\mathrm{ml} \times 100 \mathrm{~g}^{-1} \mathrm{~min}^{-1}\right)$} & Whole hemisphere & $22.26 \pm 10.98$ & $31.94 \pm 23.28$ & $44.33 \pm 22.64$ & $68.00 \pm 57.24$ \\
\hline & Basal ganglion & $27.56 \pm 19.90$ & $38.46 \pm 36.92$ & $52.76 \pm 24.91$ & $85.05 \pm 69.01$ \\
\hline & White matter & $10.43 \pm 8.05$ & $15.38 \pm 27.16$ & $18.58 \pm 9.67$ & $25.39 \pm 23.70$ \\
\hline \multirow[t]{3}{*}{ MTT (second) } & Whole hemisphere & $10.47 \pm 4.31$ & $9.03 \pm 3.27$ & $5.85 \pm 1.84$ & $4.75 \pm 4.74$ \\
\hline & Basal ganglion & $9.74 \pm 9.80$ & $6.98 \pm 5.83$ & $5.02 \pm 1.77$ & $3.49 \pm 1.42$ \\
\hline & White matter & $16.10 \pm 10.17$ & $11.89 \pm 8.73$ & $7.79 \pm 5.73$ & $6.17 \pm 3.16$ \\
\hline
\end{tabular}

\section{Results}

Patient characteristics

One hundred and six patients were retrospectively identified that matched our study inclusion criteria. Six patients were excluded because of significant motion artifact on the PCT series. One hundred patients remained that constituted our study population (56 males, 44 females, median age 65 , range 18-91). Fifty-eight patients had ischemia on the right side and 42 patients had ischemia on the left. Seventy-one patients had middle cerebral artery strokes, 21 patients had anterior cerebral artery strokes, and 9 patients had posterior cerebral artery strokes. Twenty-seven patients received intravenous tPA, and 36 patients received endovascular revascularization therapy. Fifty-four patients showed recanalization, and 46 patients showed persistent arterial occlusion. Final infarct volume was measured at a median time of 26 days (interquartile range 7.3-70), and delineated on MRI in 66 patients and CT in 34 patients.

Comparison of delay-sensitive and delay-insensitive CBV, $\mathrm{CBF}$, and MTT values

There was no difference in terms of CBV values between delay-sensitive and delay-insensitive deconvolution. This was expected as CBV is calculated as an indicator of partial volume averaging from the area of the time-density curves in each voxel of the image compared to a reference "venous" voxel [6]. CBV values are not influenced by the selection of the arterial input function and the type of deconvolution used.

For whole-hemisphere measurements, delay-sensitive MTT measurements were significantly greater than delayinsensitive MTT values $(p<0.05)$, and delay-sensitive CBF values were significantly smaller than delay-insensitive CBF values $(p<0.05)$ (Tables 3 and 4, Fig. 1). Similar observations were made in the basal ganglia and in the white matter. The higher the CBF or the MTT values, the greater the difference between delay-sensitive and delayinsensitive deconvolution. This explains why the difference was more marked in the nonischemic tissue for CBF (higher normal values) and in the ischemic tissue for MTT (higher ischemic values).

Comparison of delay-sensitive and delay-insensitive volumes of ischemic core and penumbra

Ischemic core and penumbra volumes calculated from delayinsensitive deconvolution using MTT $135 \%$ and CBV $2 \mathrm{ml} \times$ $100 \mathrm{~g}^{-1}$ thresholds (ischemic core $33.51 \pm 30.56 \mathrm{ml}$, ischemic penumbra $58.90 \pm 35.49 \mathrm{ml}$, ischemic volume $92.43 \pm$

Table 4 Discrepancy ( $\Delta$ = delay-insensitive - delay-sensitive) between delay-insensitive and delay-sensitive deconvolution for the whole hemisphere, basal ganglion, and the white matter measurements of CBF and MTT separately, for the ischemic side and nonischemic side

\begin{tabular}{|c|c|c|c|c|c|c|c|c|c|}
\hline \multirow[t]{2}{*}{ Variable } & \multirow[t]{2}{*}{ Location } & \multicolumn{4}{|c|}{ Ischemic side } & \multicolumn{4}{|c|}{ Nonischemic side } \\
\hline & & Mean & $95 \% \mathrm{CI}$ & Slope & $p$ & Mean & $95 \% \mathrm{CI}$ & Slope & $p$ \\
\hline \multirow[t]{3}{*}{$\mathrm{CBF}$} & Whole hemisphere & 8.9 & 6.2 to 11.5 & 0.65 & $<0.001$ & 19.621 & 14.5 to 24.7 & 0.69 & $<0.001$ \\
\hline & Basal ganglion & 11.0 & 6.7 to 15.4 & 0.63 & $<0.001$ & 27.845 & 21.1 to 34.6 & 0.74 & $<0.001$ \\
\hline & White matter & 3.5 & 0.9 to 6.2 & 1.57 & 0.009 & 5.536 & 3.2 to 7.8 & 0.81 & $<0.001$ \\
\hline \multirow[t]{3}{*}{ MTT } & Whole hemisphere & -2.3 & -3.1 to -1.5 & -0.63 & $<0.001$ & -1.008 & -1.9 to -0.1 & -0.68 & 0.044 \\
\hline & Basal ganglion & -2.7 & -4.3 to -1.1 & -0.64 & 0.001 & -1.498 & -1.8 to -1.2 & -0.58 & $<0.001$ \\
\hline & White matter & 1.9 & -6.1 to -1.3 & -0.86 & 0.003 & -11.411 & -2.6 to -0.6 & -0.75 & 0.002 \\
\hline
\end{tabular}


Fig. 1 Bland-Altman plots demonstrating the relationship between delay-sensitive $\mathrm{CBF}$ and $\Delta$ = delay-insensitive CBF delay-sensitive $\mathrm{CBF}$ and between delay-sensitive MTT and $\Delta=$ delay-insensitive MTT - delaysensitive MTT. Dashed line denotes 0 difference. Solid line denotes the regression line of best fit. Delay-sensitive MTT measurements were significantly greater than delay-insensitive MTT values $(p<0.05)$, and delaysensitive CBF values were significantly smaller than delayinsensitive CBF values $(p<0.05)$

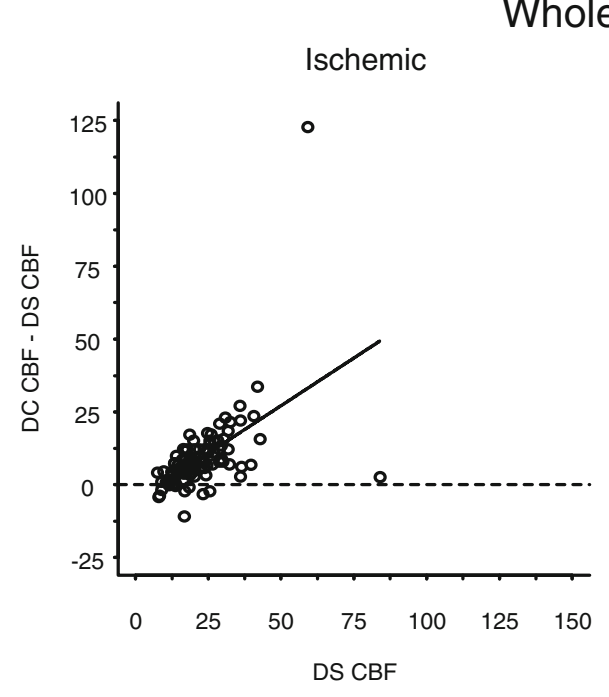

Non-Ischemic

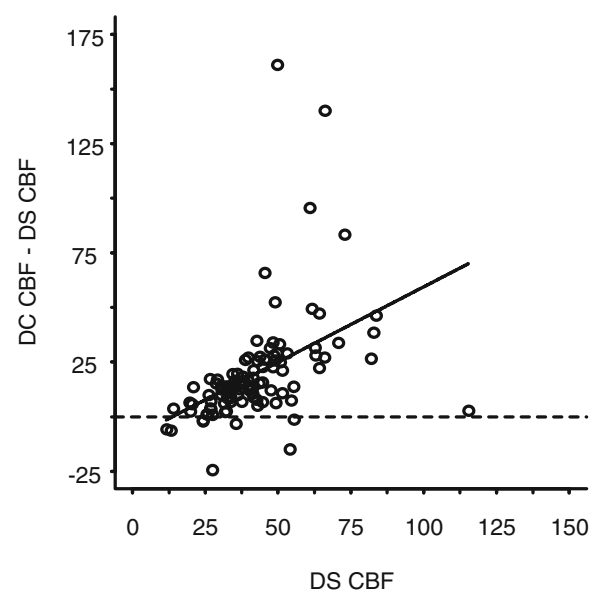

Ischemic

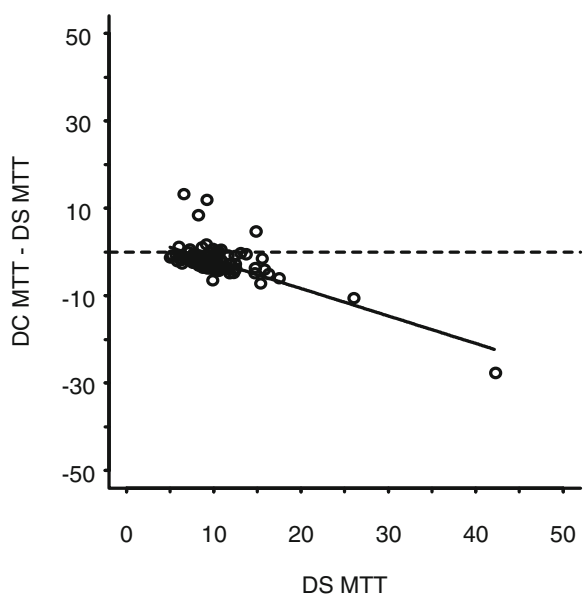

Non-Ischemic

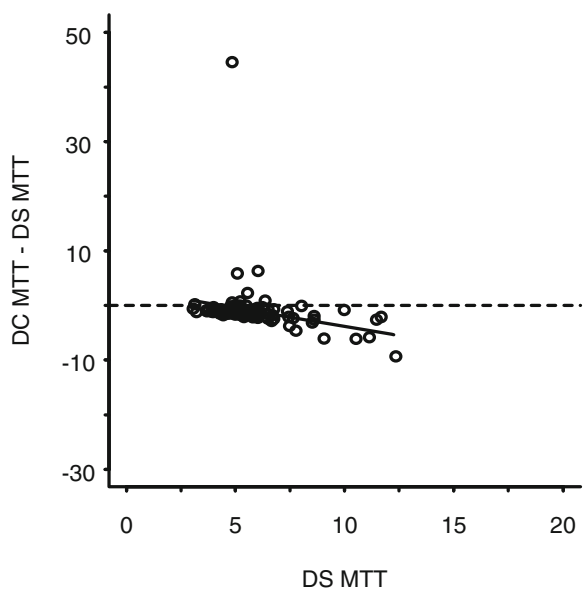

Table 5 Discrepancy ( $\Delta$ = delay-insensitive - delay-sensitive) between delay-insensitive and delay-sensitive deconvolution for the volumes of ischemic core, ischemic penumbra, and total ischemic volume. These volumes for delay-sensitive deconvolution were calculated using MTT threshold of $145 \%$ of the contralateral side values to define PCT ischemic area, and CBV threshold of $2.0 \mathrm{ml} / 100 \mathrm{~g}$ to define PCT ischemic penumbra

\begin{tabular}{|c|c|c|c|c|c|c|c|c|c|c|}
\hline \multirow{2}{*}{$\begin{array}{l}\text { Delay-insensitive } \\
\text { MTT threshold (\%) }\end{array}$} & \multirow{2}{*}{$\begin{array}{l}\text { Delay-insensitive CBV } \\
\text { threshold }\left(\mathrm{ml} \times 100 \mathrm{~g}^{-1}\right)\end{array}$} & \multicolumn{3}{|c|}{ Ischemic core volume (ml) } & \multicolumn{3}{|c|}{ Ischemic penumbra volume $(\mathrm{ml})$} & \multicolumn{3}{|c|}{ Ischemic volume (ml) } \\
\hline & & Mean & $95 \% \mathrm{CI}$ & $p$ & Mean & $95 \% \mathrm{CI}$ & $p$ & Mean & $95 \% \mathrm{CI}$ & $p$ \\
\hline 135 & 2 & -3.4 & -7.3 to 0.6 & 0.091 & 6.1 & 1.2 to 11.1 & 0.15 & 2.7 & -0.7 to 6.1 & 0.113 \\
\hline 145 & 2 & -6.6 & -10.7 to -2.5 & 0.002 & 0.9 & -3.8 to -5.7 & 0.690 & -5.7 & -9.4 to -2.0 & 0.003 \\
\hline 155 & 2 & -9.7 & -14.0 to -5.2 & $<0.001$ & -4.1 & -8.7 to 0.6 & 0.086 & -13.5 & -17.8 to -9.2 & $<0.001$ \\
\hline 165 & 2 & -12.5 & -17.1 to -7.8 & $<0.001$ & -7.9 & -12.5 to -3.3 & 0.001 & -20.3 & -25.3 to -15.4 & $<0.001$ \\
\hline 135 & 1.9 & -7.4 & -11.3 to -3.4 & $<0.001$ & 11.2 & 6.2 to 16.2 & $<0.001$ & 3.8 & 0.5 to 7.2 & 0.026 \\
\hline 145 & 1.9 & -10.1 & -14.3 to -5.9 & $<0.001$ & 4.6 & -0.1 to 9.3 & 0.051 & -5.5 & -9.1 to -1.9 & 0.003 \\
\hline 155 & 1.9 & -12.9 & -17.3 to -8.4 & $<0.001$ & -0.6 & -5.2 to 3.9 & 0.777 & -13.5 & -17.8 to -9.2 & $<0.001$ \\
\hline 165 & 1.9 & -15.3 & -19.9 to -10.5 & $<0.001$ & -4.9 & -9.6 to -0.3 & 0.038 & -20.2 & -25.1 to -15.3 & $<0.001$ \\
\hline 125 & 1.8 & -8.5 & -12.5 to -4.5 & $<0.001$ & 22.5 & 16.7 to 28.3 & $<0.001$ & 14.0 & 9.9 to 18.1 & $<0.001$ \\
\hline 135 & 1.8 & -11.3 & -15.4 to -7.2 & $<0.001$ & 14.4 & 9.3 to 19.6 & $<0.001$ & 3.1 & -0.6 to 6.8 & 0.104 \\
\hline 145 & 1.8 & -13.8 & -18.1 to -9.5 & $<0.001$ & 8.1 & 3.4 to 12.9 & $<0.001$ & -5.6 & -9.2 to -2.0 & 0.003 \\
\hline
\end{tabular}


$51.98 \mathrm{ml}$ ) were not significantly different $(p=0.091-0.150)$ from ischemic core and penumbras volume calculated from delay-sensitive deconvolution using MTT $145 \%$ and CBV $2 \mathrm{ml} \times 100 \mathrm{~g}^{-1}$ validated thresholds (ischemic core $36.93 \pm$ $31.96 \mathrm{ml}$, ischemic penumbra $52.89 \pm 31.88 \mathrm{ml}$, ischemic volume $89.82 \pm 49.98 \mathrm{ml}$ ) (Table 5, Fig. 2).
Comparison of delay-sensitive and delay-insensitive modified aspect scores for ischemic core and penumbra

The modified ASPECT scores for the ischemic core obtained from delay-sensitive and delay-insensitive deconvolution (Table 6) showed a concordance of $89 \%$ (95\% confidence
Fig. 2 Graphical illustration of the differences between delaysensitive and delay-insensitive deconvolution. The same PCT data in the same patient with a right middle cerebral artery ischemic stroke was twice, using each of the two methods. CBV values do not differ between the two methods. MTT values are higher, and $\mathrm{CBF}$ values lower, with delay-sensitive deconvolution. However, with the appropriate, different thresholds (145\% MTT, CBV $2 \mathrm{ml} \times$ $100 \mathrm{~g}^{-1} \mathrm{~min}^{-1}$ for delay-sensitive deconvolution, and $135 \%$ MTT, CBV $2 \mathrm{ml} \times 100 \mathrm{~g}^{-1} \mathrm{~min}^{-1}$ for delay-insensitive deconvolution), the two methods yield very similar results in terms of prediction of the ischemic core (red) and penumbra (green) volumes

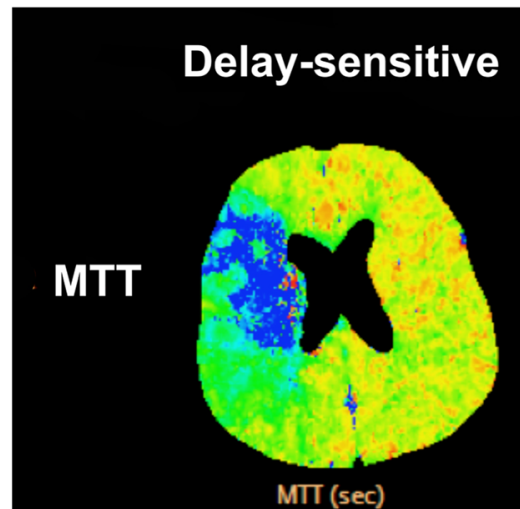

\section{Delay-corrected}
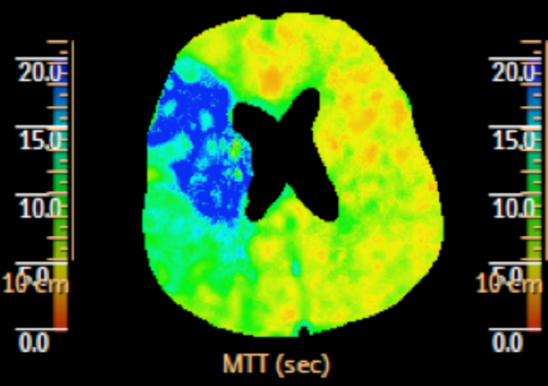

CBF

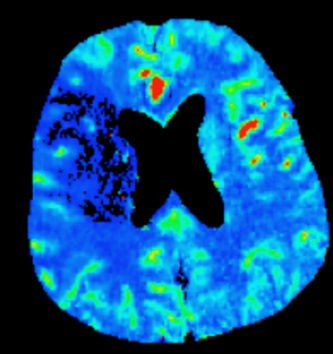

$\mathrm{CBF}(\mathrm{ml} / 100 \mathrm{~g} / \mathrm{min})$
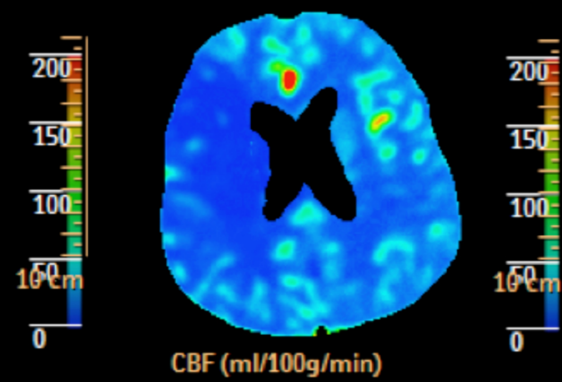

CBV

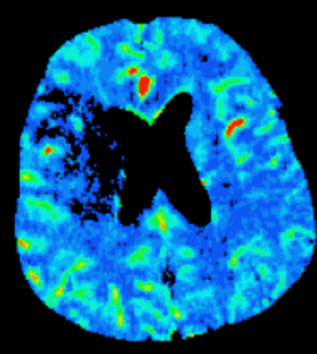

$\operatorname{CBV}(\mathrm{ml} / 100 \mathrm{~g})$
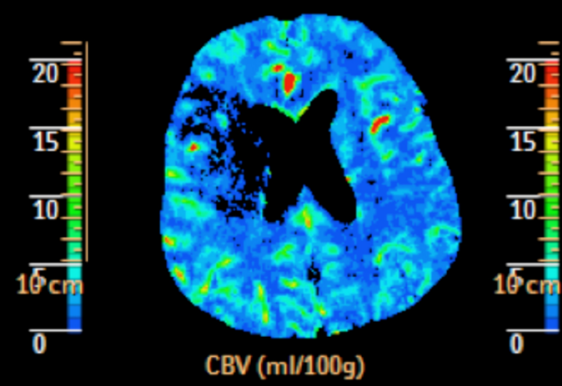

\section{infarct}
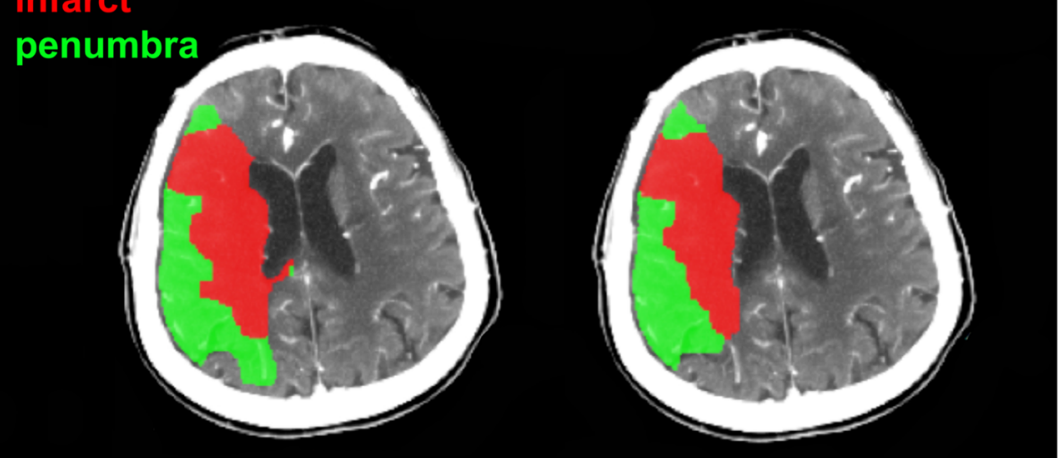
Table 6 ASPECT score concordance for the ischemic core between delay-sensitive and delay-insensitive deconvolution

\begin{tabular}{llll}
\hline Ischemic core & & Delay-sensitive \\
& & $0-7$ & $8-14$ \\
Delay-insensitive & $0-7$ & 80 & 7 \\
& $8-14$ & 4 & 9 \\
Ischemic penumbra & & Delay-sensitive & \\
Delay-insensitive & & $0-7$ & $8-14$ \\
& $0-7$ & 76 & 8 \\
& $8-14$ & 5 & 11 \\
\hline
\end{tabular}

interval 81-94\%) and were not significantly different from each other (McNemar $p=0.366)$.

The modified ASPECT scores for the ischemic penumbra obtained from delay-sensitive and delay-insensitive deconvolution (Table 6) showed a concordance of $87 \%$
(95\% confidence interval 79-93\%) and were not significantly different from each other (McNemar $p=0.405$ ).

Assessment of tissue fate of delay-sensitive and delay-insensitive ischemic core and penumbra

In patients with persistent occlusion, the total ischemic areas (core + penumbra) defined on delay-sensitive and delayinsensitive deconvolution both correlated similarly with the final infarct volume on the slices matching the initial PCT (linear regression coefficients of 0.99 for delay-sensitive deconvolution and 1.09 for delay-insensitive deconvolution, correlation coefficients of 0.809 for delay-sensitive deconvolution and 0.794 for delay-insensitive deconvolution, Fig. 2).

In patients with recanalization, the ischemic core defined on delay-sensitive and delay-insensitive deconvolution both
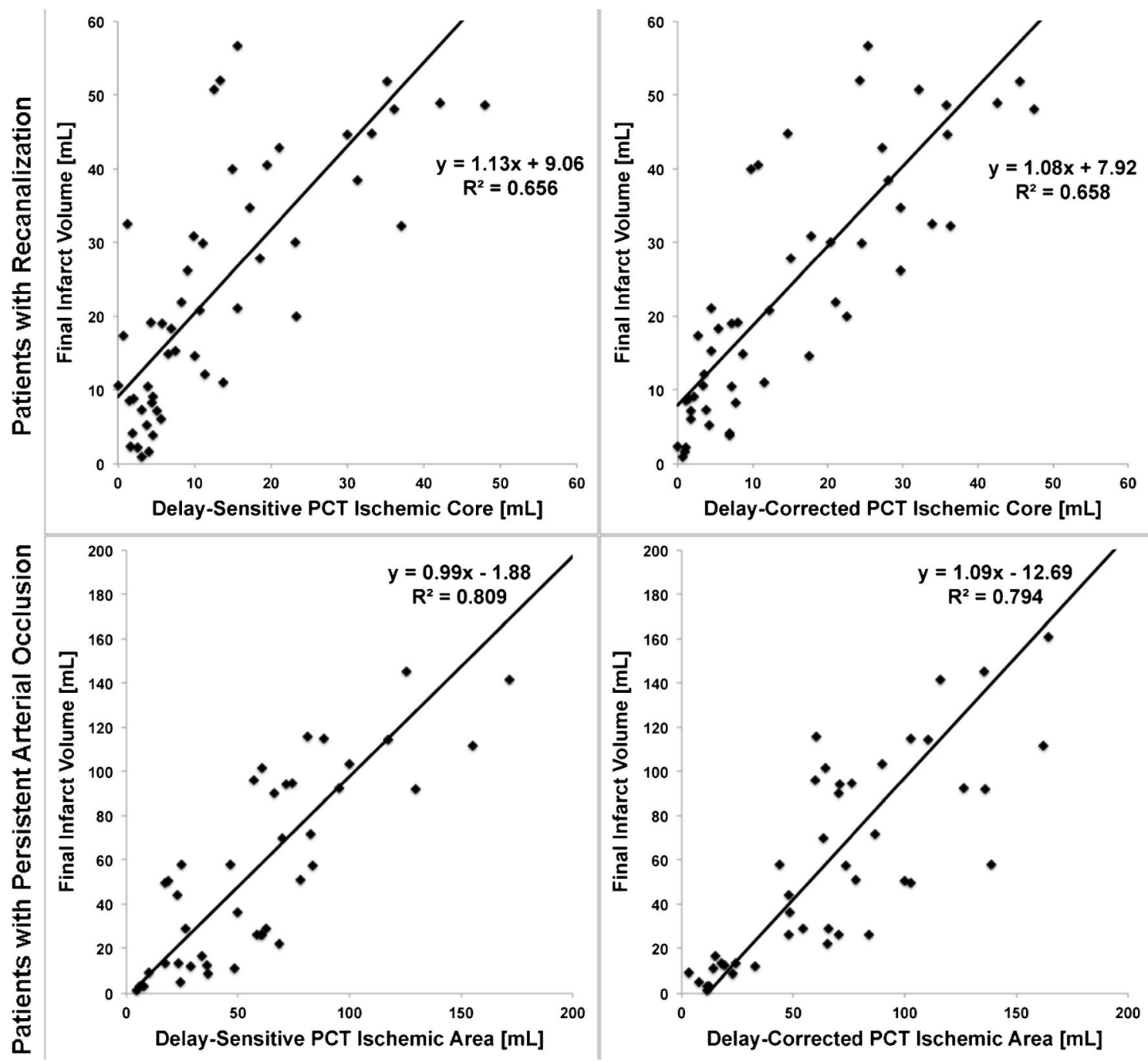

Fig. 3 Comparison of the final infarct volume with the delay-sensitive and delay-insensitive ischemic core (in patients with recanalization) and ischemic core + penumbra (in patients with persistent arterial occlusion) 
correlated similarly with the final infarct volume on the slices matching the initial PCT (linear regression coefficients of 1.13 for delay-sensitive deconvolution and 1.08 for delayinsensitive deconvolution, correlation coefficients of 0.656 for delay-sensitive deconvolution and 0.658 for delayinsensitive deconvolution, Fig. 3). Of note, results were similar for 16- and 64-slice CT scanners.

\section{Discussion}

The goal of this article was to compare delay-sensitive and delay-insensitive deconvolution for PCT processing in patients with acute ischemic stroke. We found that $\mathrm{CBV}$ values were not affected by the type of deconvolution used for PCT processing. MTT values were higher, and CBF values lower, with delay-sensitive deconvolution compared to delayinsensitive deconvolution. However, when different, appropriate thresholds were applied to delay-sensitive (MTT $145 \%$ and CBV $2 \mathrm{ml} \times 100 \mathrm{~g}^{-1} \mathrm{~min}^{-1}$ ) and to delayinsensitive (MTT $135 \%$ and CBV $2 \mathrm{ml} \times 100 \mathrm{~g}^{-1} \mathrm{~min}^{-1}$ ) deconvolution, the resultant volumes of predicted ischemic core and penumbra were similar, as well as their anatomical distribution, and both showed similar association with final infarct volume when recanalization status was taken into account. Of note, in patients with recanalization, the final infarct tended to be underestimated by both delay-sensitive and delay-insensitive deconvolution PCT, likely because not all these patients underwent early recanalization, and the infarct grew between the time of the PCT and the time when recanalization occurred.

Our results are in agreement with previous studies. Prior MRI studies have shown that CBV values are not affected by the contrast arrival delay $[11,18]$. On the other hand, CBF and MTT values are affected by the contrast arrival delay [14]. More specifically, the contrast arrival delay in the ischemic tissue typically introduces an underestimation in the calculated CBF values and an overestimation in the calculated MTT values $[18,11]$.

The optimal thresholds to delineate the ischemic core and penumbra are different for delay-sensitive and delayinsensitive deconvolution. However, when appropriate, yet different, thresholds are applied for delay-sensitive and delay-insensitive deconvolution, the resulting prediction of ischemic core and penumbra are comparable. The thresholds we used for delay-sensitive deconvolution and as reference standards in this study were previously validated in a large prospective study [3].

We acknowledge several limitations to our study. We use one PCT processing software provided by one CT manufacturer. The identified thresholds may not work for other software packages developed by other manufacturers, including those that use Tmax thresholds [19]. However, the general conclusions of the study regarding differences and similarities between delay-sensitive and delay-insensitive deconvolution are likely to hold. Similarly, the final infarct volume was determined with a wide range and using different techniques, but the general conclusions of the study should not be affected because each dataset served as its own control for the comparison analysis between the delay-sensitive and delayinsensitive deconvolution processing methods. Finally, our study sample included different imaging protocols. Because again, each dataset served as its own control for the comparison analysis between the delay-sensitive and delayinsensitive deconvolution processing methods, the different protocols do not affect the internal validity of our study. Rather, they increase its generalizability.

In conclusion, both delay-sensitive and delay-insensitive deconvolution methods are appropriate for PCT processing in acute ischemic stroke patients. Caution should be exercised when assessing MTT and CBV values as they are affected by the deconvolution method used. Similarly, optimal thresholds to delineate ischemic core and penumbra are different, but, when appropriate different thresholds are selected for delaysensitive and delay-insensitive deconvolution, the predicted ischemic core and penumbra are similar with both methods. Thresholds need to be tailored to the specific software package in addition to the deconvolution method used.

Acknowledgments MW receives research funding from GE Healthcare and Philips Healthcare.

Ethical standards and patient consent We declare that all human and animal studies have been approved by the Institutional Review Board of the University of Virginia and have therefore been performed in accordance with the ethical standards laid down in the 1964 Declaration of Helsinki and its later amendments. We declare that all patients gave informed consent prior to inclusion in this study.

Conflict of interest We declare that we have no conflict of interest.

\section{References}

1. Shetty SK, Lev MH (2005) CT perfusion in acute stroke. Neuroimaging Clin N Am 15(3):481-501. doi:10.1016/j.nic.2005. 08.004, ix

2. Pan J, Zhang J, Huang W, Cheng X, Ling Y, Dong Q, Geng D (2013) Value of perfusion computed tomography in acute ischemic stroke: diagnosis of infarct core and penumbra. J Comput Assist Tomogr 37(5):645-649. doi:10.1097/RCT.0b013e31829866fc

3. Wintermark M, Sesay M, Barbier E, Borbely K, Dillon WP, Eastwood JD, Glenn TC, Grandin CB, Pedraza S, Soustiel JF, Nariai T, Zaharchuk G, Caille JM, Dousset V, Yonas H (2005) Comparative overview of brain perfusion imaging techniques. J Neuroradiol J Neuroradiol 32(5):294-314

4. Sviri GE, Britz GW, Lewis DH, Newell DW, Zaaroor M, Cohen W (2006) Dynamic perfusion computed tomography in the diagnosis of cerebral vasospasm. Neurosurgery 59(2):319-325. doi:10.1227/01. NEU.0000222819.18834.33, discussion 319-325 
5. Soustiel JF, Mahamid E, Goldsher D, Zaaroor M (2008) Perfusion$\mathrm{CT}$ for early assessment of traumatic cerebral contusions. Neuroradiology 50(2):189-196. doi:10.1007/s00234-007-0337-7

6. Leiva-Salinas C, Provenzale JM, Wintermark M (2011) Responses to the 10 most frequently asked questions about perfusion CT. Am J Roentgenol 196(1):53-60. doi:10.2214/Ajr.10.5705

7. Leiva-Salinas C, Wintermark M (2010) Imaging of acute ischemic stroke. Neuroimaging Clin N Am 20(4):455. doi:10.1016/J.Nic. 2010.07.002

8. Abels B, Klotz E, Tomandl BF, Kloska SP, Lell MM (2010) Perfusion CT in acute ischemic stroke: a qualitative and quantitative comparison of deconvolution and maximum slope approach. AJNR Am J Neuroradiol 31(9):1690-1698. doi:10.3174/ajnr.A2151

9. Konstas AA, Goldmakher GV, Lee TY, Lev MH (2009) Theoretic basis and technical implementations of CT perfusion in acute ischemic stroke, part 1: theoretic basis. AJNR Am J Neuroradiol 30(4): 662-668. doi:10.3174/ajnr.A1487

10. Leiva-Salinas C, Provenzale JM, Kudo K, Sasaki M, Wintermark M (2012) The alphabet soup of perfusion CT and MR imaging: terminology revisited and clarified in five questions. Neuroradiology 54(9):907-918. doi:10.1007/s00234-012-1028-6

11. Calamante F, Gadian DG, Connelly A (2000) Delay and dispersion effects in dynamic susceptibility contrast MRI: simulations using singular value decomposition. Magn Reson Med Off J Soc Magn Reson Med Soc Magn Reson Med 44(3):466-473

12. Calamante F, Yim PJ, Cebral JR (2003) Estimation of bolus dispersion effects in perfusion MRI using image-based computational fluid dynamics. NeuroImage 19(2 Pt 1):341-353

13. Wu O, Ostergaard L, Weisskoff RM, Benner T, Rosen BR, Sorensen AG (2003) Tracer arrival timing-insensitive technique for estimating flow in MR perfusion-weighted imaging using singular value decomposition with a block-circulant deconvolution matrix. Magn Reson Med Off J Soc Magn Reson Med Soc Magn Reson Med 50(1):164-174. doi:10.1002/mrm.10522

14. Schaefer PW, Mui K, Kamalian S, Nogueira RG, Gonzalez RG, Lev MH (2009) Avoiding "pseudo-reversibility" of CT-CBV infarct core lesions in acute stroke patients after thrombolytic therapy the need for algorithmically "delay-corrected" CT perfusion map postprocessing software. Stroke J Cereb Circ 40(8):2875-2878

15. Wintermark M, Maeder P, Thiran JP, Schnyder P, Meuli R (2001) Quantitative assessment of regional cerebral blood flows by perfusion CT studies at low injection rates: a critical review of the underlying theoretical models. Eur Radiol 11(7):1220-1230

16. Wintermark M, Flanders AE, Velthuis B, Meuli R, van Leeuwen M, Goldsher D, Pineda C, Serena J, van der Schaaf I, Waaijer A, Anderson J, Nesbit G, Gabriely I, Medina V, Quiles A, Pohlman S, Quist M, Schnyder P, Bogousslavsky J, Dillon WP, Pedraza S (2006) Perfusion-CT assessment of infarct core and penumbra: receiver operating characteristic curve analysis in 130 patients suspected of acute hemispheric stroke. Stroke J Cereb Circ 37(4):979-985. doi:10.1161/ 01.STR.0000209238.61459.39

17. Puetz V, Dzialowski I, Hill MD, Demchuk AM (2009) The Alberta stroke program early CT score in clinical practice: what have we learned? Int J Stroke Off J Int Stroke Soc 4(5):354-364. doi:10. $1111 / j .1747-4949.2009 .00337 . x$

18. Calamante F, Gadian DG, Connelly A (2002) Quantification of perfusion using bolus tracking magnetic resonance imaging in stroke: assumptions, limitations, and potential implications for clinical use. Stroke J Cereb Circ 33(4):1146-1151

19. Bivard A, Levi C, Spratt N, Parsons M (2013) Perfusion CT in acute stroke: a comprehensive analysis of infarct and penumbra. Radiology 267(2):543-550 